\title{
Alteration of histone $\mathrm{H3}$ lysine 9 dimethylation in peripheral white blood cells of septic patients with trauma and cancer
}

\author{
LINGHUI JIANG $^{1}$, YIQIN WANG ${ }^{2}$, DUMING ZHU ${ }^{1}$, ZHANGGANG XUE $^{1}$ and HAILEI MAO ${ }^{1}$ \\ ${ }^{1}$ Departments of Anesthesiology and Critical Care Medicine, Zhongshan Hospital, Fudan University, Shanghai 200032; \\ ${ }^{2}$ State Key Laboratory of Molecular Biology and Shanghai Key Laboratory of Molecular Andrology, Institute of Biochemistry \\ and Cell Biology, Shanghai Institutes for Biological Sciences, Chinese Academy of Sciences, Shanghai 200031, P.R. China
}

Received October 18, 2015; Accepted October 6, 2016

DOI: $10.3892 / \mathrm{mmr} .2016 .5958$

\begin{abstract}
The present study aimed to investigate the clinical significance of histone methylation in sepsis. A total of 43 blood samples from trauma and esophageal cancer patients with or without sepsis were collected. Immunofluorescence staining of isolated peripheral white blood cells (WBCs) was conducted. Co-stained 293T cells served as a reference, to allow the levels of histone methylation in different types of WBCs from patients to be determined. Immunostaining analyses revealed different levels of histone 3 lysine 9 dimethylation (H3K9me2) in neutrophils (Neu), lymphocytes (Lym), and monocytes (Mon) from trauma patients. Compared with trauma patients, the levels of $\mathrm{H} 3 \mathrm{~K} 9 \mathrm{me} 2$ were elevated in the three types of WBCs from cancer patients. When combined with sepsis, trauma patients demonstrated increased $\mathrm{H} 3 \mathrm{~K} 9 \mathrm{me} 2$ levels in Neu ( $\mathrm{P}=0.0005)$ and Mon ( $\mathrm{P}=0.0002)$, whereas cancer patients had a significant decrease of $\mathrm{H} 3 \mathrm{~K} 9 \mathrm{me} 2$ levels in the three types of WBCs (Neu, $\mathrm{P}=0.0003$; Lym, $\mathrm{P}=0.007$; Mon, $\mathrm{P}=0.007)$. The $\mathrm{H} 3 \mathrm{~K} 9 \mathrm{me} 2$ alterations in patients with trauma and cancer were different with the occurrence of sepsis. A larger cohort study is warranted to explore the diagnostic significance and prognostic implications of altered histone methylation in septic patients.
\end{abstract}

Correspondence to: Dr Hailei Mao or Dr Zhanggang Xue, Departments of Anesthesiology and Critical Care Medicine, Zhongshan Hospital, Fudan University, 180 Fenglin Road, Shanghai 200032, P.R. China

E-mail: mao.hailei@zs-hospital.sh.cn

E-mail: xue.zhanggang@zs-hospital.sh.cn

Abbreviations: H3K9me2, histone 3 lysine 9 dimethylation; WBCs, white blood cells; Neu, neutrophils; Lym, lymphocytes; Mon, monocytes; CRP, C-reactive protein; APACHE II, Acute Physiology and Chronic Health Evaluation II; SOFA, Sequential Organ Failure Assessment; AUC, area under the curve

Key words: histone 3 lysine 9 dimethylation, peripheral white blood cells, immunofluorescence staining, sepsis, trauma, esophageal cancer

\section{Introduction}

Sepsis is a systemic inflammatory response syndrome caused by infection, which may lead to multiple organ dysfunction. It is the leading cause of mortality in critically ill patients (1). The inflammatory response is primarily mediated by white blood cells (WBCs), including neutrophils (Neu), lymphocytes (Lym), and monocytes (Mon) $(2,3)$. Previous studies have suggested that the inflammatory response may be regulated by histone methylation (4-9).

Histone methylation regulates transcription and chromatin dynamics, and has roles in pathogenic development and the maintenance of normal physiology $(10,11)$. Methylation occurs on lysine and arginine residues of histone proteins (12). Each lysine may undergo three different states of methylation, with one (mono), two (di), or three (tri) potential methyl groups being covalently attached to the amine group of the lysine side chain, and arginine may be mono- or di-methylated symmetrically or asymmetrically $(13,14)$. Histone methylation may repress or activate transcription, depending on the specific residues and modification states. Histone 3 lysine 9 (H3K9), H3K27 and H4K20 methylation are associated with transcriptional repression, whereas methylation of H3K4, H3K36 and H3K79 are associated with transcriptional activation (15-17).

Histone methylation is a reversible process, catalyzed by two antagonistic groups of enzymes, histone methyltransferases (HMTs) and demethylases (HDMs), with the levels of each of these determining the biological outcome (18-21). Certain studies have revealed that the process of inflammation and infection in inflammatory cells is regulated by histone methylation, including $\mathrm{H} 3 \mathrm{~K} 4, \mathrm{H} 3 \mathrm{~K} 9$ and $\mathrm{H} 3 \mathrm{~K} 27$ methylation (4-9,22-24). Specific HMTs or HDMs were revealed to link histone methylation to transcriptional genes of inflammation/infection, including nuclear factor $\kappa B$ (NF- $\kappa \mathrm{B}$ ), tumor necrosis factor- $\alpha$ and interleukin-6, when inflammatory cells, particularly Mon or macrophages, were investigated in vitro $(4-9,22-24)$. The majority of studies that have been performed examined $\mathrm{H} 3 \mathrm{~K} 9$ methylation, and $\mathrm{H} 3 \mathrm{~K} 9$ dimethylation (H3K9me2) is primarily associated with the inflammatory response (4-9,22-24). However, the specific histone methylation alterations that occur in the peripheral WBCs of patients with critical illness and sepsis remain to be elucidated. 
The present study hypothesized that histone methylation in peripheral WBCs from patients is important in sepsis occurrence and progression. The current study established and optimized an immunofluorescence staining method to investigate $\mathrm{H} 3 \mathrm{~K} 9 \mathrm{me} 2$ alterations in WBCs from critically ill patients. The pilot data provided clinical evidence for the hypothesis of histone methylation in WBCs, and suggested that the characteristic changes of histone methylation are a potential diagnostic and prognostic biomarker for septic patienthowever, further evaluation in a larger cohort is required.

\section{Materials and methods}

Study design. The present study was approved by the Research Ethics Board of Zhongshan Hospital, Fudan University (Shanghai,China).Written informed consent was obtained from all recruited patients. The majority of patients admitted to the surgical intensive care unit (ICU) of Zhongshan Hospital were suffering from postoperative trauma or esophageal cancer, and were frequently affected by sepsitherefore, these two groups of patients became the focus of the present study. To minimize individual differences, each group comprised a similar number of patients within the same age range. Only male patients were enrolled to avoid gender bias. A total of 43 patients (23 trauma and 20 esophageal canceage range, 40-75 years) were enrolled in the present study between October 2012 and December 2014. The enrolled patients were divided into four groups: i) Trauma, ii) trauma+sepsis, iii) cancer and iv) cancer+sepsis. Exclusion criteria were: Patients with trauma and cancer, endocrine disorders (including diabetes and hyperthyroidism), obesity [body weight $20 \%$ greater than the ideal standard, which is equal to height (cm)-105], or any other chronic organ impairments. None of the cases had infectious diseases for more than two weeks prior to the sample collection.

Traumatic insults resulted from road accidents, accidental traumas and falls. The pathological diagnosis of all enrolled esophageal cancer patients was squamous cell carcinoma, grade II-III. Sepsis patients (12 trauma; 11 postoperative esophageal cancer) were diagnosed according to the 2001 International Sepsis Definition Conference (25). The clinical and biochemical parameters of the subjects were recorded, including number, age and gender, Acute Physiology and Chronic Health Evaluation II (APACHE II) score (26), Sequential Organ Failure Assessment (SOFA) score (27), microbiological findings, site of infection, length of ICU stay, mortality occurring in ICU, C-reactive protein (CRP), procalcitonin and predominant variables of routine blood tests.

Sample collection and preparation. Blood samples were collected within the first $24 \mathrm{~h}$ following operations in nonseptic patients, and following diagnosis of sepsis in septic patients. The blood was obtained using an existing arterial or venous catheter and transferred into a sterile heparin anticoagulatant vacutainer. Healthy volunteers donated $5 \mathrm{ml}$ blood samples via the elbow vein. The samples were processed within $2 \mathrm{~h}$ following collection.

Samples were centrifuged at $1,200 \times \mathrm{g}$ for $15 \mathrm{~min}$ at $4^{\circ} \mathrm{C}$ and the plasma supernatant was collected for storage. Red blood cell lysis buffer ( $3 \mathrm{ml}$; Beyotime Institute of Biotechnology, Haimen, China) was added, and the blood cell samples were mixed at room temperature for $10 \mathrm{~min}$, centrifuged at $1,000 \mathrm{x} g$ for 2 min at $4^{\circ} \mathrm{C}$, and the supernatant discarded. WBC samples were washed with PBS buffer 2-3 times. 293T cells (human embryonic kidney cells, kindly granted by Prof. Charlie Degui Chen, Shanghai Institutes for Biological Sciences, Chinese Academy of Sciences, China) were grown in Iscove's DMEM containing in $10 \%$ fetal calf serum medium for $48 \mathrm{~h}$. An aliquot of WBCs and 293T cells in a ratio of 4:1 were mixed and resuspended in PBS. Following this, $10 \mu \mathrm{l}$ of the mixed cell suspension $\left(\sim 1 \times 10^{4}\right.$ cells) was dropped vertically onto a slide. Following drying of the slide, the cells were fixed in $4 \%$ paraformaldehyde for $10 \mathrm{~min}$, and washed with PBS 2-3 times. Finally, the slides were stored at $-80^{\circ} \mathrm{C}$ until measurement.

Western blot analysis. Proteins from the prepared WBCs were extracted using SDS lysis buffer (Beyotime Institute of Biotechnology). To examine H3K9me2 levels in WBCs, a $10 \mu \mathrm{g}$ extract of cell proteins from these cells was separated on a $15 \%$ SDS-PAGE gel. The proteins were transferred to a polyvinyl difluoride membrane in blocking reagent [5\% milk in Tris-buffered saline (TBST)] at room temperature for $30 \mathrm{~min}$. The membrane was washed with $1 \mathrm{X}$ TBST three times. A mouse histone H3K9me2-specific antibody (1:2,000; cat. no. ab1220; Abcam, Cambridge, MA, USA) was used for western blot analysis, and a rabbit histone $\mathrm{H} 3$ antibody (1:5,000; cat. no. ab1791; Abcam) served as a loading control. The primary antibodies were incubated with the membrane at room temperature for $90 \mathrm{~min}$. The membrane was then washed with $1 \mathrm{X}$ TBST three times, and incubated with goat anti-mouse and goat anti-rabbit horseradish peroxidase-conjugated secondary antibodies (1:10,000; cat. nos. 115-035-003 and 111-035-045; Jackson ImmunoResearch Laboratories, Inc., West Grove, PA, USA) at room temperature for $60 \mathrm{~min}$. Enhanced chemiluminescence (SuperSignal West Pico Chemiluminescent Substrate; Thermo Fisher Scientific, Inc., Waltham, MA, USA) was subsequently used to visualize the blot.

Immunofluorescence staining. The stored leukocyte slides were defrosted at room temperature for $20 \mathrm{~min}$. Following washing with PBS, the cells were permeabilized with cold PBS containing $0.2 \%$ Triton X-100 for 5 min. Subsequent to blocking in $1 \%$ bovine serum albumin (Roche Diagnostics, Basel, Switzerland) in PBS at room temperature for $30 \mathrm{~min}$, the cells were incubated with a H3K9me2 antibody (1:800; cat. no. ab1220; Abcam) in a humidified chamber at $37^{\circ} \mathrm{C}$ for $60 \mathrm{~min}$, then incubated with the Cy3-labeled goat anti-mouse IgG (1:1,000; cat. no. 115-165-146; Jackson ImmunoResearch Laboratories, Inc.) at room temperature for $60 \mathrm{~min}$, and stained with DAPI, and mounted prior to viewing. Fields with appropriate cell density were selected and observed by fluorescence microscopy with the exposure time of $2 \mathrm{msec}$ for H3K9me2 and $130 \mathrm{msec}$ for DAPI. The total leukocyte cell counts in each patient sample were all $>100$ in 5 different fields.

The fluorescence intensity of different cells on the slide was analyzed by Image-Pro Plus software version 6.0 (Media Cybernetics, Inc., Rockville, MD, USA). The software allowed the staining intensity and area of each cell to be presented in one table. The quantitative intensity of the three WBC types in each sample was calculated as the mean intensity of all 
Table I. Clinical and biochemical parameters of study subjects.

\begin{tabular}{|c|c|c|c|c|}
\hline Variable & Trauma & Trauma+sepsis & Cancer & Cancer+sepsis \\
\hline Primary etiology & \multicolumn{2}{|c|}{ Trauma } & \multicolumn{2}{|c|}{ Esophageal squamous cell carcinoma } \\
\hline Number & 11 & 12 & 9 & 11 \\
\hline Gender & \multicolumn{4}{|c|}{ Male } \\
\hline Age (years) & $52.46 \pm 17.17$ & $62.17 \pm 18.47$ & $56.11 \pm 7.29$ & $62.55 \pm 9.34$ \\
\hline APACHE II score & $2.64 \pm 2.11$ & $16.58 \pm 6.58^{\mathrm{b}}$ & $3.56 \pm 1.24$ & $15.27 \pm 7.07^{\mathrm{b}}$ \\
\hline SOFA score & $0.45 \pm 0.69$ & $7.33 \pm 4.89^{b}$ & $1.33 \pm 0.87$ & $8.36 \pm 5.90^{\mathrm{b}}$ \\
\hline \multicolumn{5}{|l|}{ Infection } \\
\hline Gram-positive bacteria & NA & 5 & NA & 4 \\
\hline Gram-negative bacteria & NA & 3 & NA & 6 \\
\hline \multicolumn{5}{|l|}{ Site of infection } \\
\hline Pulmonary & NA & 8 & NA & 8 \\
\hline Abdominal & NA & 5 & NA & 3 \\
\hline Other & NA & 0 & NA & 1 \\
\hline Patients with organ insufficiency & 0 & 6 & 0 & 7 \\
\hline Length of ICU stay (days) & $1 \pm 0$ & $21.33 \pm 14.25^{\mathrm{b}}$ & $9.11 \pm 7.36$ & $15.63 \pm 9.23$ \\
\hline Mortality occurring in ICU & 0 & 4 & 0 & 2 \\
\hline WBC $\left(\times 10^{9} / 1\right)$ & $7.31 \pm 2.03$ & $12.27 \pm 4.67^{\mathrm{b}}$ & $13.63 \pm 3.52$ & $14.84 \pm 4.96$ \\
\hline Neu $(\%)$ & $62.75 \pm 7.15$ & $87.27 \pm 5.27^{\mathrm{b}}$ & $83.13 \pm 2.30$ & $88.78 \pm 3.39^{\mathrm{b}}$ \\
\hline $\operatorname{Lym}(\%)$ & $28.51 \pm 7.91$ & $7.62 \pm 4.56^{\mathrm{b}}$ & $7.49 \pm 3.59$ & $5.82 \pm 1.87$ \\
\hline Mon $(\%)$ & $6.53 \pm 1.96$ & $5.20 \pm 2.04$ & $7.91 \pm 1.08$ & $4.63 \pm 2.18^{b}$ \\
\hline Procalcitonin $(\mu \mathrm{g} / \mathrm{l})$ & $0.23 \pm 0.04$ & $0.17 \pm 0.07^{\mathrm{a}}$ & $0.24 \pm 0.06$ & $0.20 \pm 0.07$ \\
\hline C-reactive protein (mg/l) & $9.75 \pm 21.23$ & $154.07 \pm 109.64^{\mathrm{b}}$ & $40.95 \pm 27.84$ & $91.74 \pm 77.18^{\mathrm{a}}$ \\
\hline
\end{tabular}

${ }^{\mathrm{a}} \mathrm{P}<0.05$ and ${ }^{\mathrm{b}} \mathrm{P}<0.01$ vs. non-sepsis patients. Data are expressed as the mean \pm standard deviation. NA, not applicable; APACHE II, acute physiology and chronic health evaluation; SOFA, sequential organ failure assessment; WBC, white blood cells; Neu, neutrophils; Lym, lymphocytes; Mon, monocytes.

observed cells. All data were analyzed by two independent investigators, and the intensity values quantified individually were averaged by calculation of the mean to obtain the final intensity. Microsoft Excel software 2007 (Microsoft Corporation, Redmond, WA, USA) was used to calculate the fluorescence intensity of different cells.

Statistical analysis. The data were analyzed using the unpaired Student's t-test for comparison of two groups, and one way analysis of variance for data from all four groups followed by the Bonferroni post-hoc test. All analyses were performed using Stata software version 10.0MP (StataCorp LP, College Station, TX, USA). Data are presented as the mean \pm standard deviation. $\mathrm{P}<0.05$ was considered to indicate a statistically significant difference.

\section{Results}

Patient characteristics. Table I presents a comparison of characteristics between non-septic and septic trauma $(n=23)$ and esophageal cancer $(n=20)$ patients. Routine blood tests revealed a normal WBC count in patients in the trauma group; however, levels of $\mathrm{Neu}$ and Mon in the other three groups were elevated, along with a reduced percentage of Lym. It was demonstrated that there were significant differences in the percentages of increased Neu and decreased Lym between sepsis and non-sepsis trauma patients. Additionally, the level of CRP was elevated in sepsis compared with non-sepsis grouphowever, this was not the case for procalcitonin (Table I).

Western blot analysis. The present study conducted a western blot analysis of the total level of histone methylation in whole WBCs in sepsis and non-sepsis patients. Notably, H3 from each sample contained three bands, with the largest band corresponding to the intact $\mathrm{H} 3$ probed with histone $\mathrm{H} 3 \mathrm{~K} 9 \mathrm{me} 2$ antibody (data not shown), suggesting that the two smaller bands were derived from cleavage of histone H3 following $\mathrm{K} 9$ residues in WBCs. $\mathrm{H} 3$ cleavage following residues A21 to $\mathrm{K} 27$ in mouse embryonic stem cells has been reported previously $(28,29)$, suggesting that the cleaved N-terminal fragments, including A1-A21, A1-A23 and A1-A27, containing the target $\mathrm{K} 9$ residue, escaped detection. The present study therefore failed to obtain the total level of histone H3K9me2 from whole WBCs by western blot analysis. The levels of histone methylation from specific types of leukocytes were not identified or compared.

Immunofluorescence staining of WBCs. To examine the level of histone methylation in different WBCs, the present study used an immunofluorescence staining method. Considering 
A

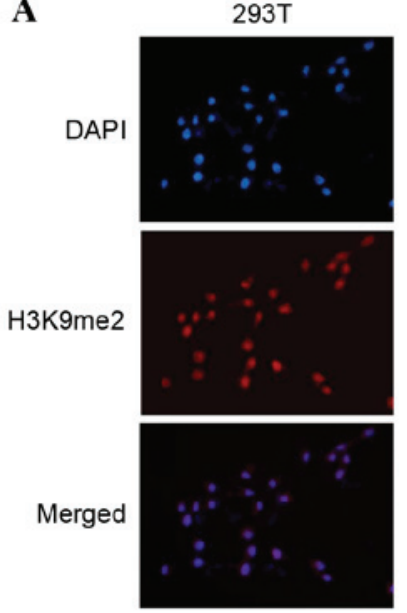

B
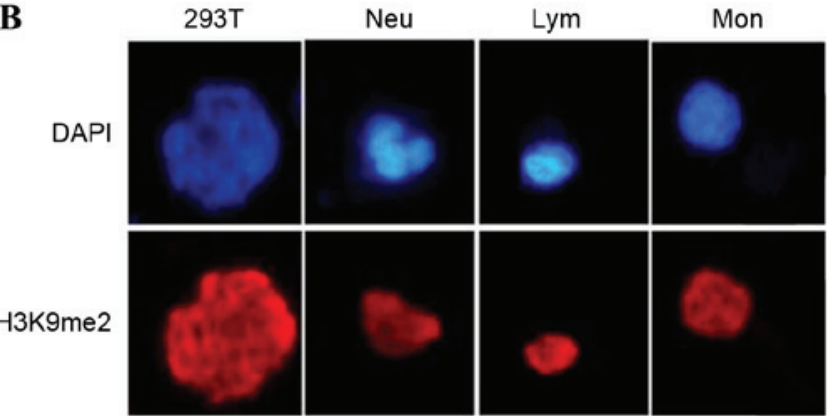

Merged
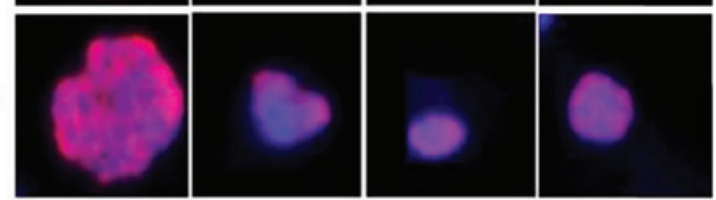

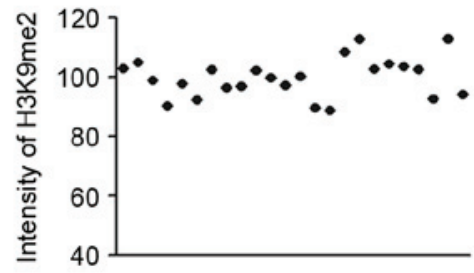

Figure 1. Immunofluorescence staining of H3K9me2 in 293T cells and three types of WBCs. (A) Homogenous fluorescence staining and intensity of H3K9me2 in $293 \mathrm{~T}$ cells; DAPI was used to stain the nuclei. The scatter plot indicates that $293 \mathrm{~T}$ cells demonstrated homogeneous staining. (B) Different nuclear morphology of 293T and three types of WBCs, Neu, Lym and Mon allows distinction of the four cell types. H3K9me2, histone 3 lysine 9 dimethylation; WBCs, white blood cells; Neu, neutrophils; Lym, lymphocytes, Mon, monocytes.

the complex cell components, wide range of cell number, and short survival time in vitro of peripheral WBCs, the leukocytes were directly separated within $2 \mathrm{~h}$ following blood collection to obtain the maximum number of live cells. Various suspension solvents and fixed slides were used to analyze leukocytes, due to complications in fixing the suspended WBCs onto a glass slide, and the suspension, blood smear and drop methods were compared. It was concluded that use of fresh leukocyte samples, PBS for cell suspension and the drop method enabled optimal immunofluorescence staining of WBCs.

To control for the influence of different staining operations and compare the immunofluorescence between samples, 293T cells were co-stained alongside WBCs, and served as a reference. 293T cells have stable and homogeneous staining intensity (Fig. 1A), and contain nuclei with a shape distinct to the three primary types of WBCs, Neu, Lym and Mon. Neu has a lobulated or rod-shaped nucleus with loose chromatin; Lym has a round or oval nucleus with dense chromatin; and Mon is larger than Lym, and has a kidney- or horseshoe-shaped nucleus with loose chromatin. The nuclei of $293 \mathrm{~T}$ cells are irregular, round and significantly larger than those of WBCs (Fig. 1B).

Sepsis-induced alterations of $\mathrm{H} 3 \mathrm{~K} 9 \mathrm{me} 2$ in WBCs from trauma and cancer patients. Immunostaining analysis detected different levels of histone $\mathrm{H} 3 \mathrm{~K} 9 \mathrm{me} 2$ in the three types of WBCs from trauma patients. The staining intensity of Lym was stronger than that of Neu and Mon (Fig. 2). The levels of $\mathrm{H} 3 \mathrm{~K} 9 \mathrm{me} 2$ were elevated in different WBCs from cancer patients, and demonstrated a similar level of intensity to that exhibited by 293 T cells (Fig. 3). In the trauma + sepsis group, the levels of $\mathrm{H} 3 \mathrm{~K} 9 \mathrm{me} 2$ in all of the three WBCs increased $\sim 20 \%$, this was particularly observed for $\mathrm{Neu}(\mathrm{P}=0.0005)$ and Mon ( $\mathrm{P}=0.0002$; Figs. 2 and 4). Notably, the presence of sepsis significantly reduced the elevation of $\mathrm{H} 3 \mathrm{~K} 9 \mathrm{me} 2$ in cancer patients by 20 to $40 \%$ (Figs. 3 and 4). The serum CRP values from trauma and cancer patients were elevated when sepsis was present; however, there was no statistical significance indicated in the cancer patients (Fig. 4).

Differential H3K9me2 expression in WBCs. The intensity of $\mathrm{H} 3 \mathrm{~K} 9 \mathrm{me} 2$ in WBCs between non-septic patients with different underlying diseases, trauma and cancer, was compared. An increase of histone methylation in cancer patients was demonstrated, in the absence or presence of sepsis. However, the elevated histone methylation in the three WBCs in cancer and septic trauma varied. The H3K9me2 levels of Lym and Mon cells in cancer patients were similar compared with trauma patients with sepsis, whereas the levels in Neu cells were greater (Fig. 4).

These results indicated that immunofluorescence staining may be used to compare histone methylation in different WBCs. Sepsis in trauma and cancer patients had opposing effects on histone methylation; sepsis increased H3K9me2 levels in trauma patients and decreased them in cancer.

Diagnostic significance of H3K9me2 in WBCs for sepsis. Furthermore, the H3K9me2 levels were used as a diagnostic marker for sepsis using a receiver operating characteristic analysis (Fig. 5). The diagnostic significance was evaluated by the area under the curve (AUC). The AUC values of H3K9me2 in the three WBCs from trauma patients were 0.70-0.73; these 

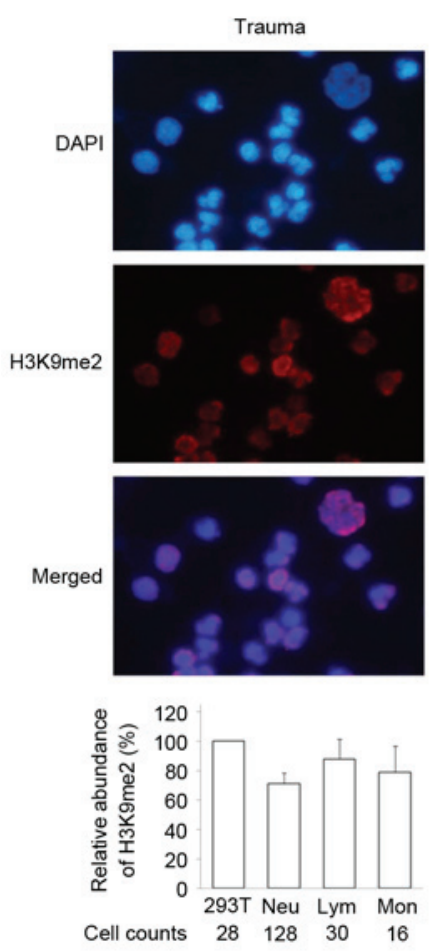
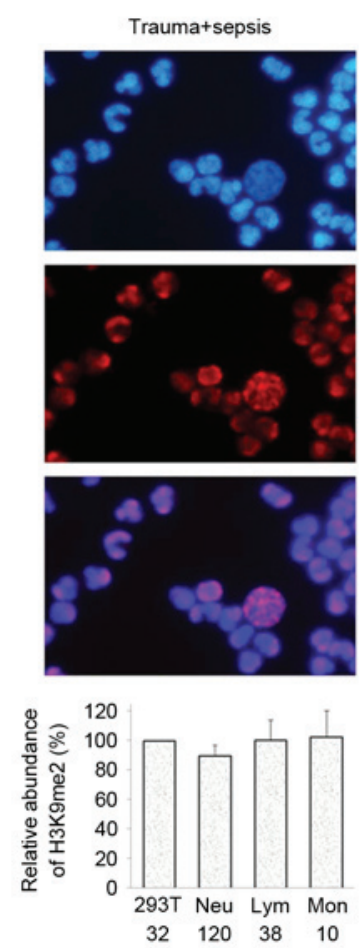

Figure 2. Immunofluorescence staining of $\mathrm{H} 3 \mathrm{~K} 9 \mathrm{me} 2$ in WBCs from trauma patients. Peripheral WBCs samples were obtained from two patients, trauma and trauma+sepsis. For each sample, the average intensity of H3K9me2 in 293T cells was defined as 100 , and the relative quantitative value of $\mathrm{H} 3 \mathrm{~K} 9 \mathrm{me} 2$ in each leukocyte was calculated. The relative fluorescence abundance of $\mathrm{H} 3 \mathrm{~K} 9 \mathrm{me} 2$ in counted Neu, Lym and Mon cells is presented as the mean \pm standard deviation. $\mathrm{H} 3 \mathrm{~K} 9 \mathrm{me} 2$, histone 3 lysine 9 dimethylation; WBCs, white blood cells; Neu, neutrophils; Lym, lymphocytes, Mon, monocytes.

were reduced compared with the 0.98 demonstrated by CRP, indicating a poor predictive value of $\mathrm{H} 3 \mathrm{~K} 9 \mathrm{me} 2$ for the occurrence of sepsis in trauma patients. By contrast, in cancer patients, the AUC values of $\mathrm{H} 3 \mathrm{~K} 9 \mathrm{me} 2$ in the three WBCs were 0.88 for Neu, 0.84 for Lym and 0.80 for Mon, all greater than the 0.67 demonstrated by CRP (Fig. 5). A cutoff of 93.79 in Neu yielded $100 \%$ sensitivity and 66\% specificity, 97.15 in Lym demonstrated $90 \%$ sensitivity and $77 \%$ specificity, and 86.05 in Mon demonstrated $81 \%$ sensitivity and $66 \%$ specificity for the occurrence of sepsis in cancer patients. Considering the percentage of Neu of total WBCs, H3K9me2 in WBCs exhibited a unique diagnostic significance for the occurrence of sepsis in cancer patients compared with the commonly used indicator.

\section{Discussion}

Previous studies have suggested that the inflammatory response in sepsis is regulated by histone methylation, including H3K4, H3K 9 and H3K27 methylation $(4,9)$. However, clinical evidence is lacking. The present study verified that the levels of histone methylation of peripheral WBCs were altered in critically ill patients.
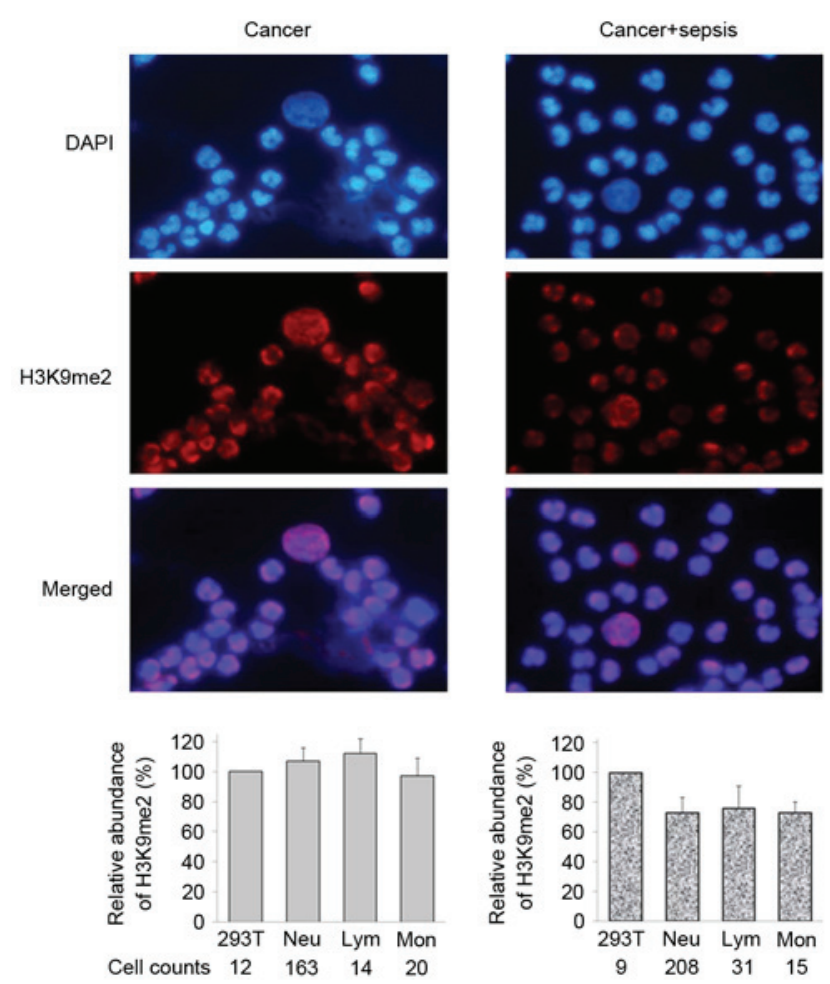

Figure 3. Immunofluorescence staining of $\mathrm{H} 3 \mathrm{~K} 9 \mathrm{me} 2$ in WBCs from cancer patients. Peripheral WBCs samples were obtained from two patients, cancer and cancer+sepsis. For each sample, the average intensity of H3K9me2 in $293 \mathrm{~T}$ cells was defined as 100 , and the relative quantitative value of $\mathrm{H} 3 \mathrm{~K} 9 \mathrm{me} 2$ in each leukocyte was calculated. The relative fluorescence abundance of $\mathrm{H} 3 \mathrm{~K} 9 \mathrm{me} 2$ in counted Neu, Lym and Mon cells is presented as the mean \pm standard deviation. $\mathrm{H} 3 \mathrm{~K} 9 \mathrm{me} 2$, histone 3 lysine 9 dimethylation; WBCs, white blood cells; Neu, neutrophils; Lym, lymphocytes, Mon, monocytes.

The present study established and optimized an immunofluorescence staining method for WBCs. Using this method, immunofluorescence intensity in different leukocytes was simultaneously determined, and the levels of histone methylation from different patient samples were compared, with co-stained 293T cells serving as a reference. The results of the present study indicated that this method is applicable to a wider range of cellular targets. The data suggested that sepsis increased the levels of $\mathrm{H} 3 \mathrm{~K} 9 \mathrm{me} 2 \mathrm{in}$ all three types of WBCs from trauma patients. The gene-specific silencing methylation is a key feature of the endotoxin tolerant phenotype demonstrated in a THP-1 human Mon model of severe systemic inflammation (SSI) and in blood polymorphonuclear leukocytes of patients with SSI $(6,8,24,30,31)$. A previous study suggested that the NF- $\mathrm{kB}$ component RelB was induced by activation of endotoxin, whereas RelB induced the formation of heterochromatin via a direct effect on the histone H3K9 methyltransferase G9a, thereby inhibiting the expression of acute proinflammatory genes to generate epigenetic silencing in endotoxin tolerance (6). Additionally, the study suggested that histone H3K9 methylation was elevated during SSI, which is consistent with the results from the present study in trauma patients with sepsis. 

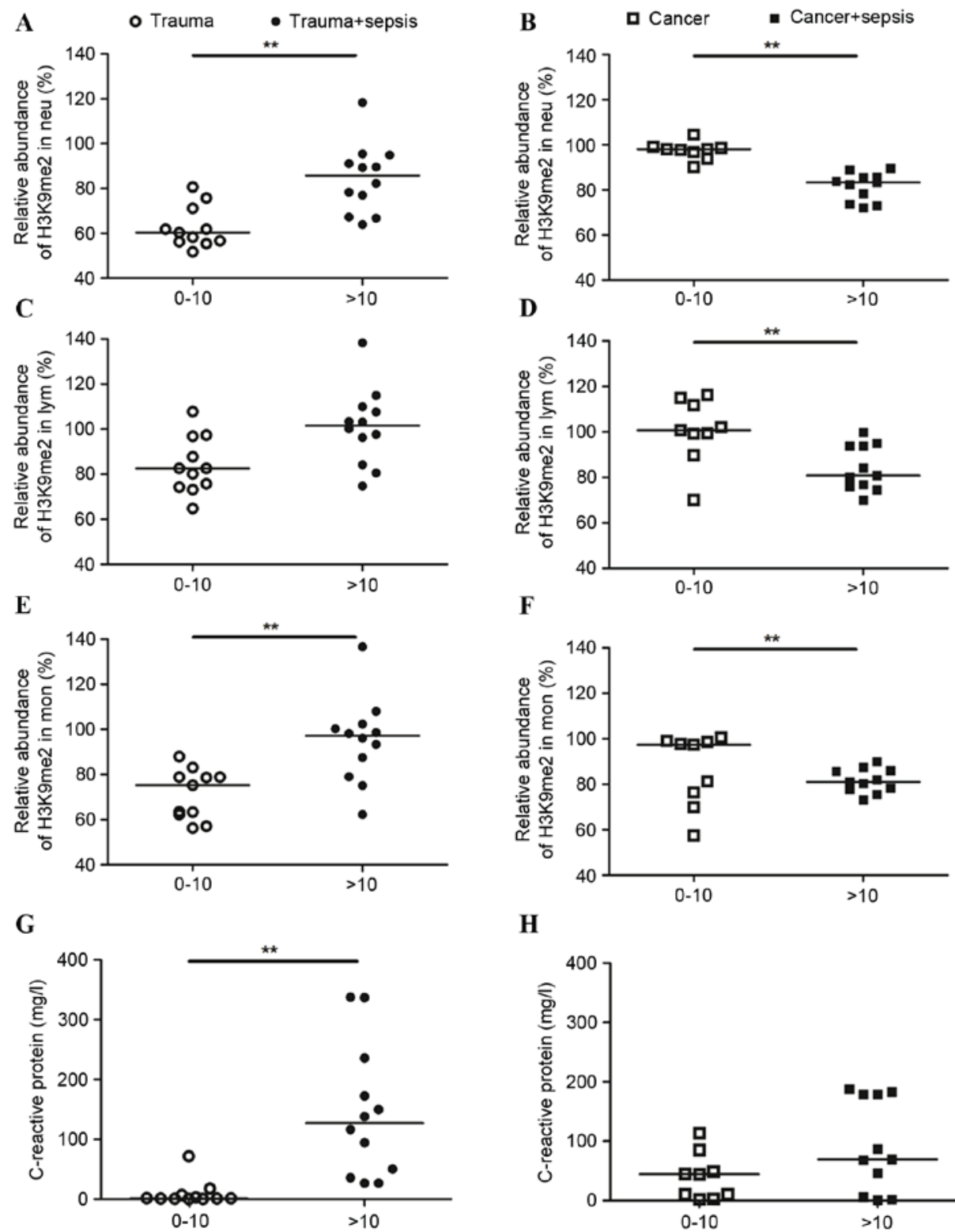

$\mathbf{H}$

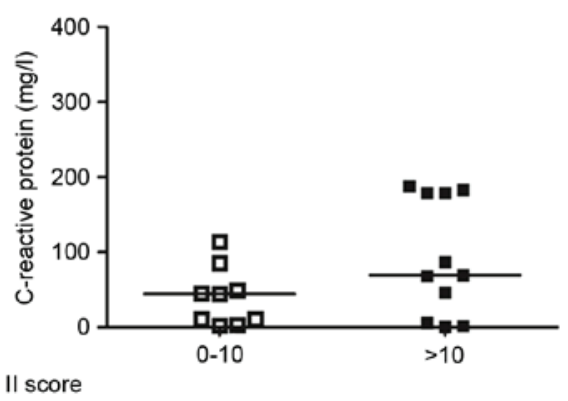

Figure 4. Association of H3K9me2 and CRP levels. H3K9me2 levels in (A and B) Neu, (C and D) Lym and (E and F) Mon, and (G and H) CRP levels in patients with trauma and cancer is presented according to APACHE II scores. The APACHE II scores of all septic patients from trauma and cancer groups were $>10$, whereas the scores of all patients without sepsis were between 0 and 10. Sepsis in trauma and cancer patients had opposing effects on histone methylation; sepsis increased H3K9me2 levels in trauma patients and decreased them in cancer. ${ }^{* *} \mathrm{P}<0.01$. H3K9me2, histone 3 lysine 9 dimethylation; APACHE II, Acute Physiology and Chronic Health Evaluation II; CRP, C-reactive protein; Neu, neutrophils; Lym, lymphocytes; Mon, monocytes.

By contrast, the occurrence of sepsis significantly downregulated $\mathrm{H} 3 \mathrm{~K} 9$ methylation in the three WBC types from esophageal cancer patients, which was elevated in the cancer group by $20-40 \%$ compared with the trauma group. The current study compared the levels of $\mathrm{H} 3 \mathrm{~K} 9 \mathrm{me} 2$ in WBCs from three healthy volunteers and trauma patients by immunostaining analyses, and no marked difference was observed. These results suggest that $\mathrm{H} 3 \mathrm{~K} 9$ methylation in WBCs of cancer patients was upregulated prior to sepsis, and differently regulated in sepsis compared with trauma patients. Further research is required to elucidate an underlying mechanism.

Sepsis induced opposing effects on histone methylation in trauma and cancer patients, suggesting that differences in primary diseases should be considered. Inflammation in sepsis may not be similar in different primary diseases, with particular differences occurring in acute vs. chronic, and benign vs. malignant cases. Additionally, the potential abnormality may alter the background of the inflammatory response. Although $\mathrm{CRP}$ is a marker that is commonly used to detect inflammation or infection (25), the disordered inflammatory state associated with cancer existed prior to sepsis, reflected by the greater percentage of Neu and elevated CRP levels in the cancer group compared with the trauma group. Pre-existing inflammation reduced the predictive power of CRP for sepsis. By contrast, H3K9me2 levels in WBCs exhibited a unique diagnostic significance for sepsis in cancer patients.

The present study observed altered H3K9me2 levels in peripheral blood leukocytes from critically ill patients via 

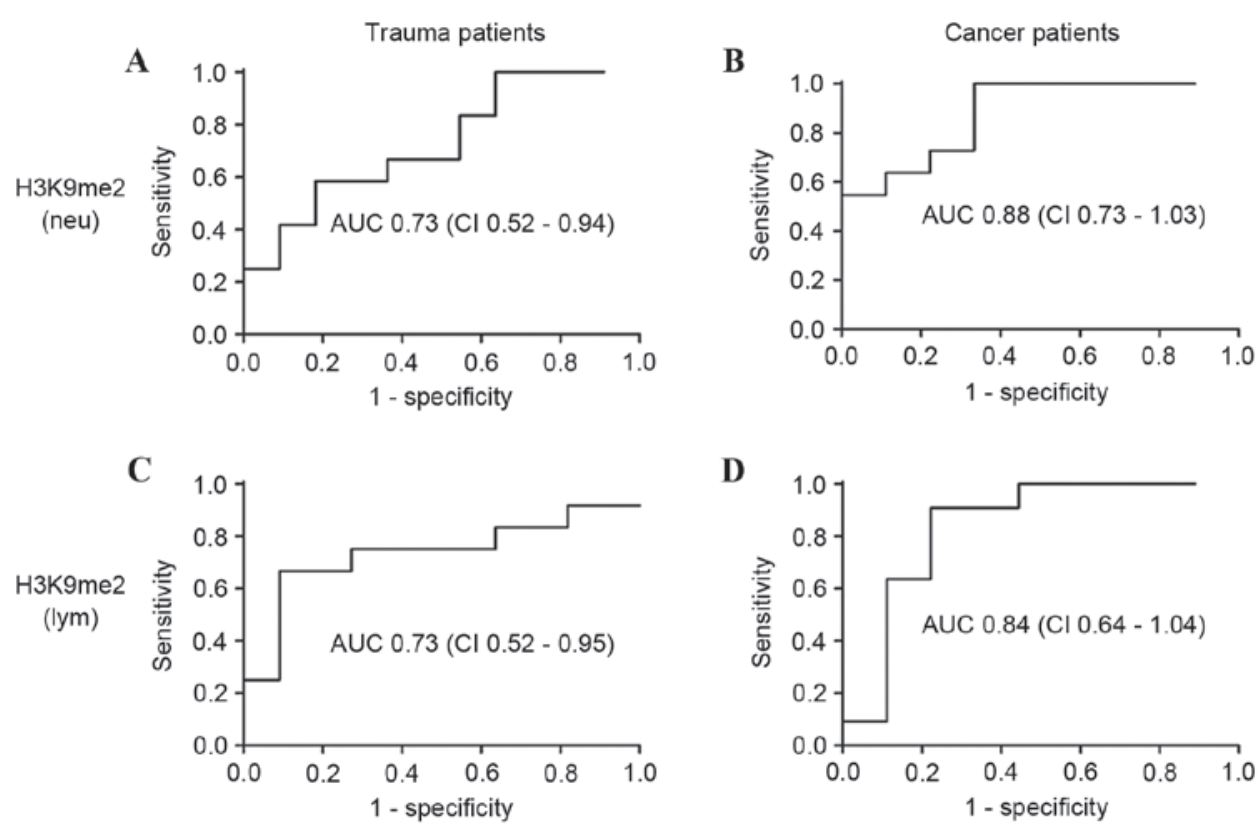

D
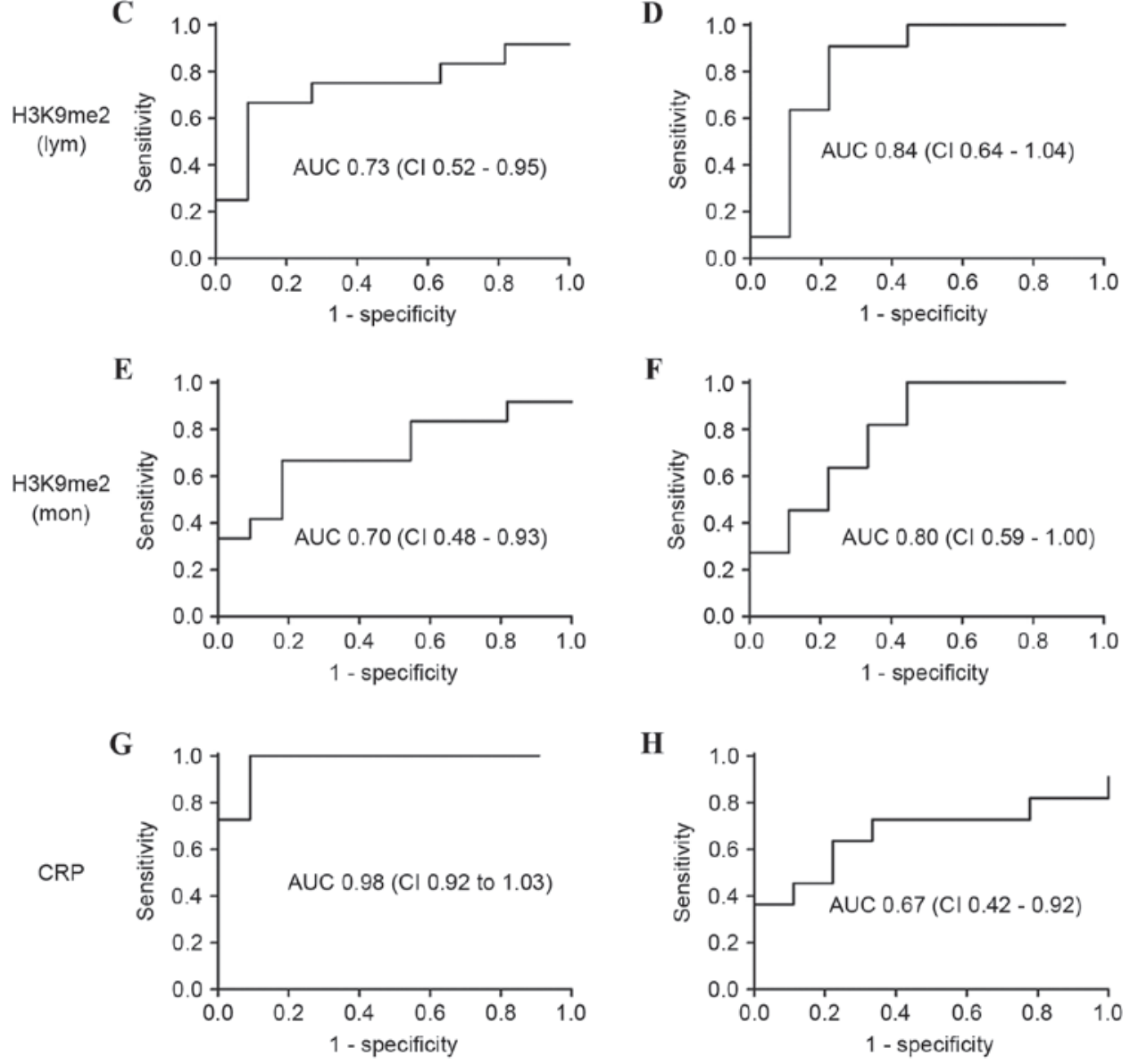

Figure 5. Receiver operating characteristic AUC of H3K9me2 and CRP levels in trauma and cancer patients. The AUC values were calculated for H3K9me2 levels in (A and B) Neu, (C and D) Lym and (E and F) Mon, and (G and H) CRP levels in patients with trauma and cancer. H3K9me2 levels had a poor predictive value for the occurrence of sepsis in trauma patients, but is a possible diagnostic marker for the occurrence of sepsis in cancer patients. AUC, area under the curve; CI, 95\% confidence interval; H3K9me2, histone 3 lysine 9 dimethylation; Neu, neutrophils; Lym, lymphocytes; Mon, monocytes; CRP, C-reactive protein.

immunofluorescence analysis, with the opposite effect induced by sepsis in trauma and cancer patients. Further studies on the differential effects of sepsis on histone methylation are currently being performed. The observation that the level of H3K9me2 is altered in sepsis is of interest, and other histone methylations, including H3K4, H3K27, H3K36, H3K79, H4K20 and arginine methylation, will be investigated in the future. It may be important to determine other potential causes of sepsis that have a similar effect on histone methylation. Establishing a unique profile of histone methylation in sepsis may enable the development of novel strategies to manage sepsis.

In conclusion, the present study analyzed histone methylation in peripheral WBCs via immunofluorescence. The
H3K9me2 levels in peripheral WBCs from trauma and cancer patients were different. Altered histone methylation in trauma and cancer patients with sepsis exhibited opposite trends, and may represent a potential diagnostic and prognostic biomarker for septic patients.

\section{Acknowledgements}

The present study was supported, in part, by the National Natural Science Foundation of China (grant no. 81101404), the Ministry of Health of China for Clinical Key Discipline in 2012 and the Grant to Young Scholarship of Fudan University (grant no. 20520133394). 


\section{References}

1. Soong J and Soni N: Sepsis: Recognition and treatment. Clin Med (Lond) 12: 276-280, 2012.

2. An G, Namas RA and Vodovotz Y: Sepsis: From pattern to mechanism and back. Crit Rev Biomed Eng 40: 341-351, 2012.

3. Cavaillon JM, Adrie C, Fitting $\mathrm{C}$ and Adib-Conquy $\mathrm{M}$ : Reprogramming of circulatory cells in sepsis and SIRS. J Endotoxin Res 11: 311-320, 2005.

4. Chen S, Ma J, Wu F, Xiong LJ, Ma H, Xu W, Lv R, Li X, Villen J, Gygi SP, et al: The histone H3 Lys 27 demethylase JMJD3 regulates gene expression by impacting transcriptional elongation. Genes Dev 26: 1364-1375, 2012.

5. De Santa F, Narang V, Yap ZH, Tusi BK, Burgold T, Austenaa L, Bucci G, Caganova M, Notarbartolo S, Casola S, et al: Jmjd3 contributes to the control of gene expression in LPS-activated macrophages. EMBO J 28: 3341-3352, 2009.

6. Chen X, El Gazzar M, Yoza BK and McCall CE: The NF-kappaB factor RelB and histone $\mathrm{H} 3$ lysine methyltransferase G9a directly interact to generate epigenetic silencing in endotoxin tolerance. J Biol Chem 284: 27857-27865, 2009.

7. De Santa F, Totaro MG, Prosperini E, Notarbartolo S, Testa G and Natoli G: The histone H3 lysine-27 demethylase Jmjd3 links inflammation to inhibition of polycomb-mediated gene silencing. Cell 130: 1083-1094, 2007.

8. Chan C, Li L, McCall CE and Yoza BK: Endotoxin tolerance disrupts chromatin remodeling and NF-kappaB transactivation at the IL-1beta promoter. J Immunol 175: 461-468, 2005.

9. Foster SL, Hargreaves DC and Medzhitov R: Gene-specific control of inflammation by TLR-induced chromatin modifications. Nature 447: 972-978, 2007.

10. Greer EL and Shi Y: Histone methylation: A dynamic mark in health, disease and inheritance. Nat Rev Genet 13: 343-357, 2012.

11. Strahl BD and Allis CD: The language of covalent histone modifications. Nature 403: 41-45, 2000.

12. Bannister AJ and Kouzarides T: Reversing histone methylation. Nature 436: 1103-1106, 2005.

13. Black JC, Van Rechem C and Whetstine JR: Histone lysine methylation dynamics: Establishment, regulation, and biological impact. Mol Cell 48: 491-507, 2012.

14. Bedford MT and Richard S: Arginine methylation an emerging regulator of protein function. Mol Cell 18: 263-272, 2005.

15. Mikkelsen TS, Ku M, Jaffe DB, Issac B, Lieberman E, Giannoukos G, Alvarez P, Brockman W, Kim TK, Koche RP, et al: Genome-wide maps of chromatin state in pluripotent and lineage-committed cells. Nature 448: 553-560, 2007.

16. Vakoc CR, Mandat SA, Olenchock BA and Blobel GA: Histone H3 lysine 9 methylation and HP1gamma are associated with transcription elongation through mammalian chromatin. Mol Cell 19: 381-391, 2005.

17. Carrozza MJ,Li B, Florens L, Suganuma T, Swanson SK, Lee KK, Shia WJ, Anderson S, Yates J, Washburn MP and Workman JL: Histone H3 methylation by Set2 directs deacetylation of coding regions by $\mathrm{Rpd} 3 \mathrm{~S}$ to suppress spurious intragenic transcription. Cell 123: 581-592, 2005.
18. Cloos PA, Christensen J, Agger K, Maiolica A, Rappsilber J, Antal T, Hansen KH and Helin K: The putative oncogene GASC1 demethylates tri- and dimethylated lysine 9 on histone H3. Nature 442: 307-311, 2006.

19. Klose RJ, Yamane K, Bae Y, Zhang D, Erdjument-Bromage H, Tempst $\mathrm{P}$, Wong $\mathrm{J}$ and Zhang $\mathrm{Y}$ : The transcriptional repressor JHDM3A demethylates trimethyl histone H3 lysine 9 and lysine 36. Nature 442: 312-316, 2006.

20. Shi Y, Lan F, Matson C, Mulligan P, Whetstine JR, Cole PA and Casero RA: Histone demethylation mediated by the nuclear amine oxidase homolog LSD1. Cell 119: 941-953, 2004.

21. Xiang S, Callaghan MM, Watson KG and Tong L: A different mechanism for the inhibition of the carboxyltransferase domain of acetyl-coenzyme A carboxylase by tepraloxydim. Proc Natl Acad Sci USA 106: 20723-20727, 2009.

22. Li Y, Reddy MA, Miao F, Shanmugam N, Yee JK, Hawkins D, Ren B and Natarajan R: Role of the histone H3 lysine 4 methyltransferase, SET7/9, in the regulation of NF-kappaB-dependent inflammatory genes. Relevance to diabetes and inflammation. J Biol Chem 283: 26771-26781, 2008.

23. Levy D, Kuo AJ, Chang Y, Schaefer U, Kitson C, Cheung P, Espejo A, Zee BM, Liu CL, Tangsombatvisit S, et al: Lysine methylation of the NF- $\kappa \mathrm{B}$ subunit RelA by SETD6 couples activity of the histone methyltransferase GLP at chromatin to tonic repression of NF-kB signaling. Nat Immunol 12: 29-36, 2011.

24. El Gazzar M, Yoza BK, Chen X, Hu J, Hawkins GA and McCall CE: G9a and HP1 couple histone and DNA methylation to TNFalpha transcription silencing during endotoxin tolerance. J Biol Chem 283: 32198-32208, 2008.

25. Levy MM, Fink MP, Marshall JC, Abraham E, Angus D, Cook D, Cohen J, Opal SM, Vincent JL and Ramsay G; SCCM/ESICM/ACCP/ATS/SIS: 2001 SCCM/ESICM/ACCP/ATS/SIS International Sepsis Definitions Conference. Crit Care Med 2003 31: 1250-1256, 2003.

26. Knaus WA, Draper EA, Wagner DP and Zimmerman JE: APACHE II: A severity of disease classification system. Crit Care Med 13: 818-829, 1985.

27. Dominguez TE and Portnoy JD: Scoring for multiple organ dysfunction: Multiple organ dysfunction score, logistic organ dysfunction, or sequential organ failure assessment. Crit Care Med 30: 1913-1914, 2002

28. Duncan EM, Muratore-Schroeder TL, Cook RG, Garcia BA, Shabanowitz J, Hunt DF and Allis CD: Cathepsin L proteolytically processes histone $\mathrm{H} 3$ during mouse embryonic stem cell differentiation. Cell 135: 284-294, 2008

29. Adams-Cioaba MA, Krupa JC, Xu C, Mort JS and Min J: Structural basis for the recognition and cleavage of histone H3 by cathepsin L. Nat Commun 2: 197, 2011.

30. El Gazzar M, Yoza BK, Hu JY, Cousart SL and McCall CE: Epigenetic silencing of tumor necrosis factor alpha during endotoxin tolerance. J Biol Chem 282: 26857-26864, 2007.

31. El Gazzar M, Yoza BK, Chen X, Garcia BA, Young NL and McCall CE: Chromatin-specific remodeling by HMGB1 and linker histone $\mathrm{H} 1$ silences proinflammatory genes during endotoxin tolerance. Mol Cell Biol 29: 1959-1971, 2009. 